\title{
On the Mean Flow Solutions of Related Rotating Disk Flows of the BEK System
}

\author{
Burhan Alveroğlu \\ Department of Mathematics, Faculty of Engineering and Natural Sciences, Bursa Technical University, 16330, Bursa, Turkey
}

\author{
Article Info \\ Keywords: Hydrodynamic stability, Ro- \\ tating flows \\ 2010 AMS: 76U05, 76E99 \\ Received: 18 September 2020 \\ Accepted: 27 November 2020 \\ Available online: 15 December 2020
}

\begin{abstract}
This paper investigates the effects of the YHP roughness model on the mean flow solutions of some flows belong to the family of the rotating BEK system flows. The governing mean flow equations are formulated in the rotating frame of reference, therefore, they include terms arising from the centrifugal force. These mean flow equations are solved using the method of lines and the backward difference method. Then, obtained results are compared for specifically selected value of roughness parameters with the results of a fundamentally different roughness model, the MW model. The results of the YHP model reveal that applying surface roughness changes the characteristics of the mean flow components. Moreover, the comparison of the YHP and MW models points that these changes are notably different for each model. Therefore, possible future researches can be conducted to investigate the stability characteristics of the flows due to the selection of the roughness model.
\end{abstract}

\section{Introduction}

The studies on the rotating disk flows have grown significantly in the literature due to common characteristics of the rotating disk flows with the swept wing flows. Both flow types have inflectional mean flow components that cause a crossflow instability known as the Type I instability mode [1,2]. The governing equations of the rotating disk flows are notably simplifier than those of the swept-wing flows due to its axisymmetric geometry. Moreover, exact similarity solutions of the Navier-Stokes equations can be found in case of the rotating disk flows [4].

Early studies on the instability properties of the rotating disk flow are established with a smooth disk configuration [4, 3]. However, the attention of the many researches have shifted to the rough disk configurations after three pioneering studies about the effect of surface roughness on the instability analysis have been published at Nature [7]-[6]. These studies have altered the common belief that rough surfaces trigger the flow instabilities and have revealed that surface roughness can be utilized to delay the onset of instabilities if the roughness is rightly sorted over the disk [5]. Therefore, determination of the right sort of roughness [5], has been a leading research field for numerous researchers over the past 30 years [8, 10, 13, 12].

An important family of rotating disk boundary layer flows are BEK system flows. This family of flows is driven by the difference of the rotation speeds of an impermeable rotating disk having an infinite radius, and the incompressible fluid rotating above the disk [4]. Both the disk and fluid rotate around the vertical axis passing through the center of disk. The angular velocities are denoted by $\Omega_{D}^{*}$ and $\Omega_{F}^{*}$ for the disk and fluid, respectively. This family includes three main types of flows: the von Kármán boundary layer flow, the Ekman boundary layer flow and the Bödewadt boundary layer flow. There are also 
infinitely many related flows between these three flows, in which the angular velocities of the disk and fluid are not equal to zero but also different.

Several attempts have been made to investigate the right sort of roughness for the BEK system flows [11, 12, 13]. In most recent studies, surface roughness has been modelled using two fundamentally different roughness models: $M W$ model developed by Miklavcicv \& Wang [14] and YHP model developed by Yoon, Hyun, and Park [15]. The first one uses partial-slip boundary conditions on the surface of the disk in order to apply roughness whereas the latter uses a fundamentally different approach. Despite easy implementation of the partial-slip boundary conditions in each direction of the disk, MW model sorts the roughness empirically. In YHP model, a new function of radial position $r$ is introduced and the roughness is modelled as a wavy surface disk using this function along with assuming a radial symmetry. Therefore, this model can sort roughness in $r$ direction only.

Many recent studies [11, 9, 12] have been conducted to determine the right sort of roughness for the BEK system flows using the YHP or MW models. The studies of Cooper et al. [12] and Garrett et al [9] are focused on von Kármán flow that is the most known member of the BEK system flows. They use both MW and YHP models, and their results reveal that two models have different effects on the instability characteristics of the flow. The study of Alveroğlu et al. [11] considered whole BEK system flows using the MW model to sort the roughness. However, the original theoretical research of the YHP model [15] conducted on the particular flows of the BEK system used a stationary frame of reference for the formulation. Therefore, it did not account the effects of the centrifugal terms appearing in a rotating frame formulation. This study uses the YHP roughness model formulated in the rotating frame and investigates the effects of the model on mean flow profiles of those particular flows of the BEK system, and compare the results with those of the previous study [11] that uses the MW model.

The overall structure of the study is stated as follows. Section 2 presents the governing steady mean-flow equations for the MW and YHP models for the entire BEK system. Computed mean flow profiles of the interested flows under the YHP model are presented in Section 3, and a comparison of the effects of the roughness models is also made in this section. Section 4 includes the conclusion.

\section{The governing mean flow equations for MW and YHP models}

The governing mean flow equations of the BEK system flows for both MW and YHP models are formulated in case of a steady fluid flow over an infinite disk. Both the disk and fluid are considered to rotate with constant angular velocities $\Omega_{D}^{*}$ and $\Omega_{F}^{*}$ around a joint axis passing through the centre of the disk. The coordinate system is assumed to be rotating with the disk The von Kármán flow occurs if $\Omega_{D}^{*} \neq 0, \Omega_{F}^{*}=0$, the Ekman flow occurs if $\Omega_{D}^{*}=\Omega_{F}^{*} \neq 0$, and the Bödewadt flow occurs if $\Omega_{D}^{*}=0, \Omega_{F}^{*} \neq 0$.

The parameter defined related to the differential rotation rate between the disk and above fluid is used to distinguish the flows of the BEK system. This parameter are defined as

$$
R o=\frac{\Omega_{F}^{*}-\Omega_{D}^{*}}{\Omega^{*}}
$$

where $\Omega^{*}$ is the system rotation rate. It is called the Rossby number. The Rossby number for the von Kármán flow is $R o=-1$ , for the Ekman flow is $R o=0$, for the Bödewadt flow is $R o=1$. Furthermore, $-1 \leq R o \leq 1$ for all the flows of the system. In this study, we will investigate the flows that occur when $-1<R o<0$.

Using a rotating coordinate frame introduces Coriolis and centrifugal terms in the equations. The Coriolis number can be defined in terms of the Rossby number as

$$
C o=2 \frac{\Omega_{D}^{*}}{\Omega^{*}}=2-R o-R o^{2}
$$

The nondimensional mean flow equations derived using the MW model are

$$
\begin{aligned}
& R o\left(U^{2}+U^{\prime} W-\left(V^{2}-1\right)\right)-C o(V-1)-U^{\prime \prime}=0 \\
& R o\left(2 U V+V^{\prime} W\right)+C o U-V^{\prime \prime}=0, \\
& R o\left(W W^{\prime}+P^{\prime}\right)-W^{\prime \prime}=0, \\
& 2 U+W^{\prime}=0 .
\end{aligned}
$$

and the boundary conditions are given by 


$$
\begin{array}{llll}
U(0)=\lambda U^{\prime}(0), & V(0)=\eta V^{\prime}(0) & \text { and } & W(0)=0, \\
U \longrightarrow 0, & V \longrightarrow 1, & \text { as } & z \longrightarrow \infty
\end{array}
$$

The full discussion on the derivation of the equations (2.2) can be found in the pioneering study of Lingwood [4]. Here, it is sufficient to note that all derivatives denoted by primes are with respect to $z$, and $U, V \& W$ are the mean flow velocity components in radial, azimuthal and axial directions, respectively. The pressure term is denoted by $P$. The boundary conditions (2.3) on the disk surface are formulated using partial-slip conditions [11, 14]. The boundary conditions at infinity are the usual no-slip conditions.

The MW model imposes the roughness over the disk surface empirically using particular values for the parameters $\eta$ and $\lambda$. The case $\lambda=\eta=0$ corresponds a smooth disk, $\eta>0, \lambda=0$ (concentric grooves) and $\eta=0, \lambda>0$ (radial grooves) correspond to anisotropic roughness, radially and azimuthally. The final case $\eta=\lambda \neq 0$ indicates an isotropic roughness. The effects of different values of these parameters on mean flow profiles of interested flows are discussed individually in the following section.

In YHP model, the governing mean flow equations are formulated in a new coordinate system $(r, \theta, \eta)$ using the Prandtl transformation [15]. Here, the new axial coordinate variable is set to $\eta=z-s(r)$, and $s(r)=\delta \cos (2 \pi r / \gamma)$ is the dimensionless surface profile.

This surface profile is axisymmetric with respect to the axis of rotation. The governing mean flow equations in this coordinate system are stated as

$$
\begin{aligned}
R o\left[r f \frac{\partial f}{\partial r}+h \frac{\partial f}{\partial \zeta}+f^{2}\left(1+r \frac{s^{\prime} s^{\prime \prime}}{1+s^{\prime 2}}\right)\right] & =\frac{R o+C o}{1+s^{\prime 2}}+\left(1+s^{\prime 2}\right) \frac{\partial^{2} f}{\partial \zeta^{2}}+\frac{g}{1+s^{\prime 2}}(C o+R o g) \\
R o\left[r f \frac{\partial g}{\partial r}+h \frac{\partial g}{\partial \zeta}\right] & =\left(1+s^{\prime 2}\right) \frac{\partial^{2} g}{\partial \zeta^{2}}-f(C o+2 g R o), \\
2 f+r \frac{\partial f}{\partial r}+\frac{\partial h}{\partial \zeta} & =0
\end{aligned}
$$

and the boundary conditions are given by

$$
\begin{aligned}
& f(r, \zeta)=g(r, \zeta)=h(r, \zeta)=0, \quad \text { at } \zeta=0, \\
& f(r, \zeta)=0, g(r, \zeta)=1 \quad \text { as } \zeta \rightarrow \infty .
\end{aligned}
$$

Here, the steady-flow profiles in radial, azimuthal and axial directions are denoted $(f, g, h)$, respectively. A full description for the derivation of those equations can be found in the previous studies [9, 15]. However, this formulation has slight modifications to the original presentation of the YHP model [15]. The Coriolis and centrifugal terms appear in this new presentation due to formulating the model in a rotating frame.

The YHP model controls the roughness using the aspect ratio parameter $a=\delta / \gamma$. For $a=0$, those equations are coincide with the previous studies of Lingwood [4] and Alveroğlu [11]. However, it is only possible to apply surface roughness only in radial direction under the YHP model due to the definition of distribution function $s(r)$. Therefore, the YHP model can be compared with the MW model in only the case of concentric grooves of roughness profile.

\section{Results and discussion}

The governing equations (2.2)-(2.3) for the MW model are the system of nonlinear ODEs and solved in the previous study of Alveroğlu [11] for different values of the parameters $\eta$ and $\lambda$ using a fourth order Runga Kutta method. However, the governing equations (2.4)-(2.5) for the YHP model are highly nonlinear system of PDEs, and we provide the solutions of the YHP model for the flows of $-1<R o<0$. In other words, we investigate the flows between the von Kármán and Ekman flows. The results arising from the YHP model for those flows are then compared with the results of the MW model.

In order to compute mean flow components those PDEs are reduced to ODEs with respect to $\zeta$ variable using method of lines. The required initial solution for the methods of lines is achieved at $r=0$ from the ODEs in $\zeta$ of the BEK system [4]. Then, the flow profiles at each incremental $r$ value are computed using the the backward difference method. The computation grid at each $r$ value is set from $\zeta=0$ to $\zeta=16$. In order to make a comparison with solutions of the MW model, the computed flow profiles of YHP model are averaged spatially. This averaged flow field is denoted by $(\bar{f}(\zeta), \bar{g}(\zeta), \bar{h}(\zeta))[9]$. 

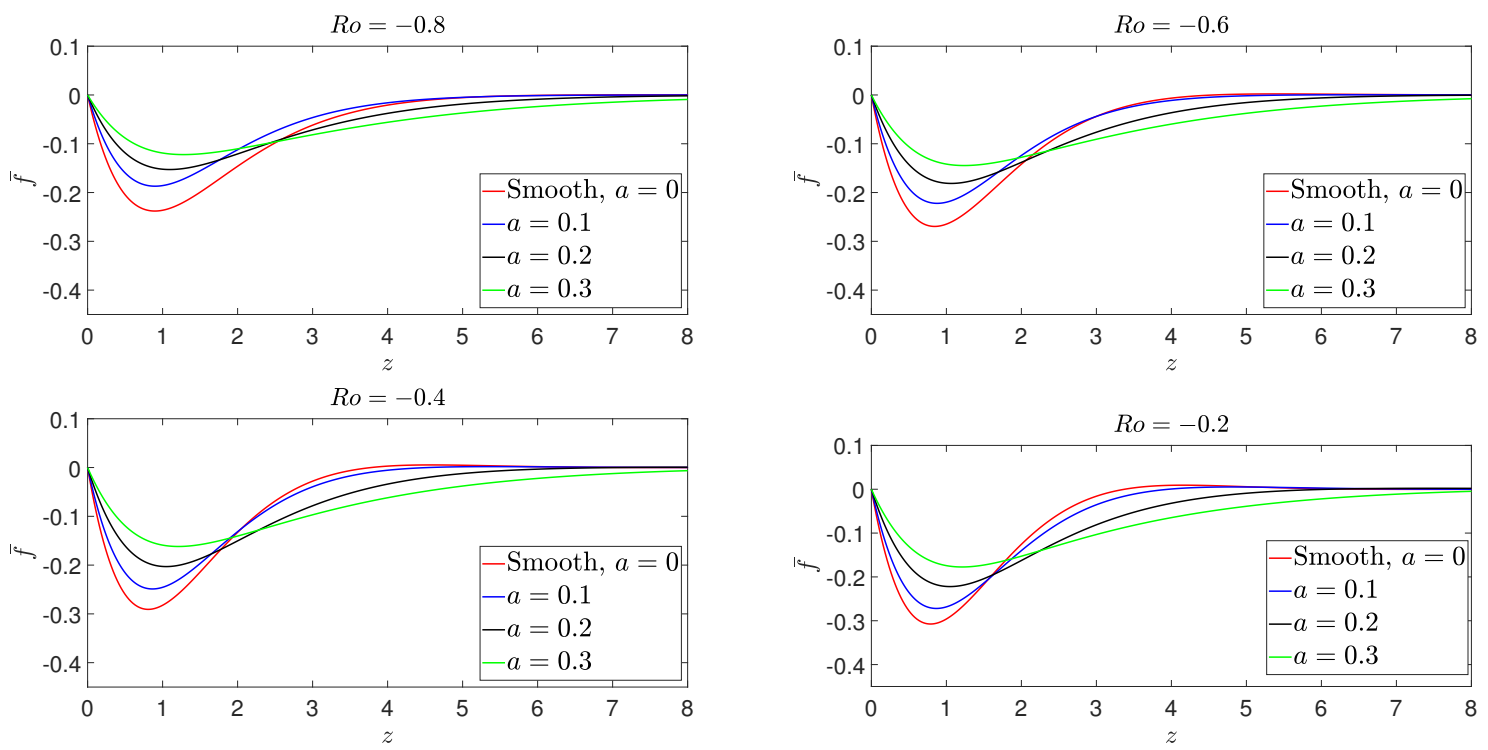

Figure 3.1: The mean flow components in radial direction at various values of roughness parameter $a$.
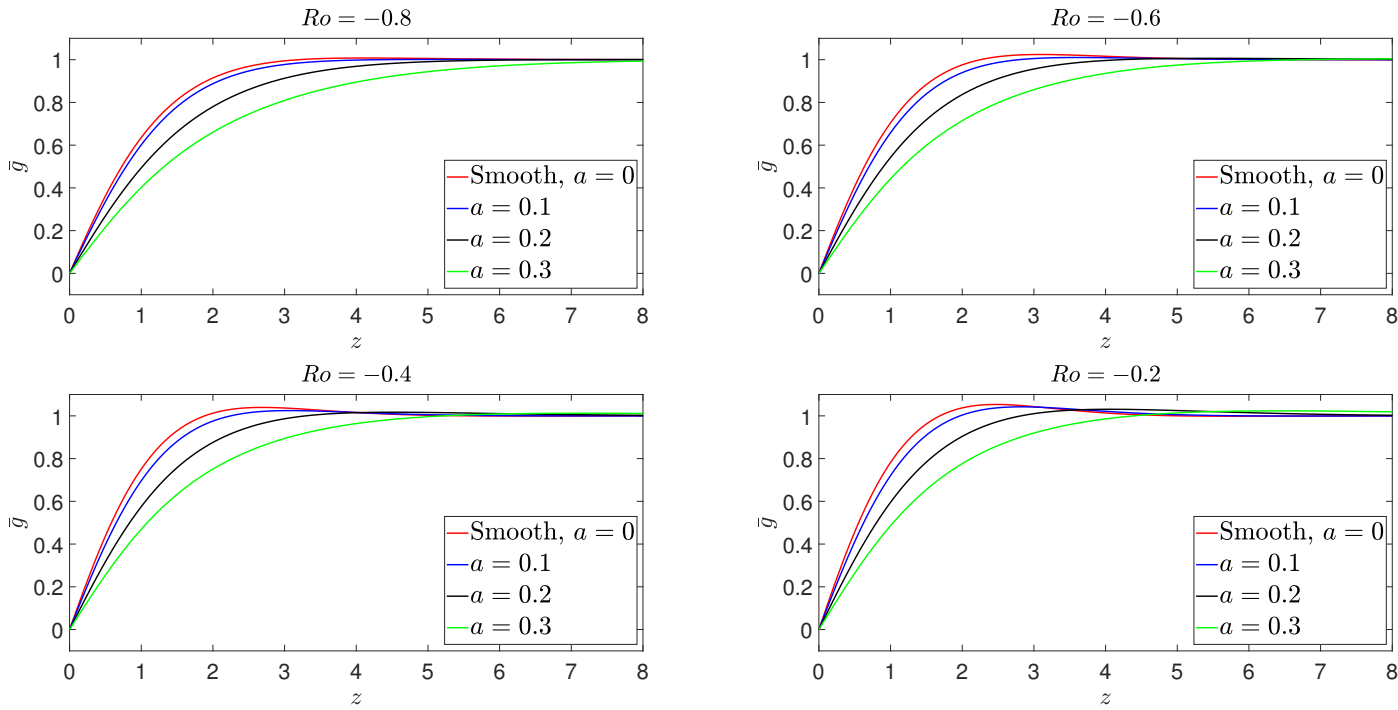

Figure 3.2: The mean flow components in azimuthal direction at various values of roughness parameter $a$.
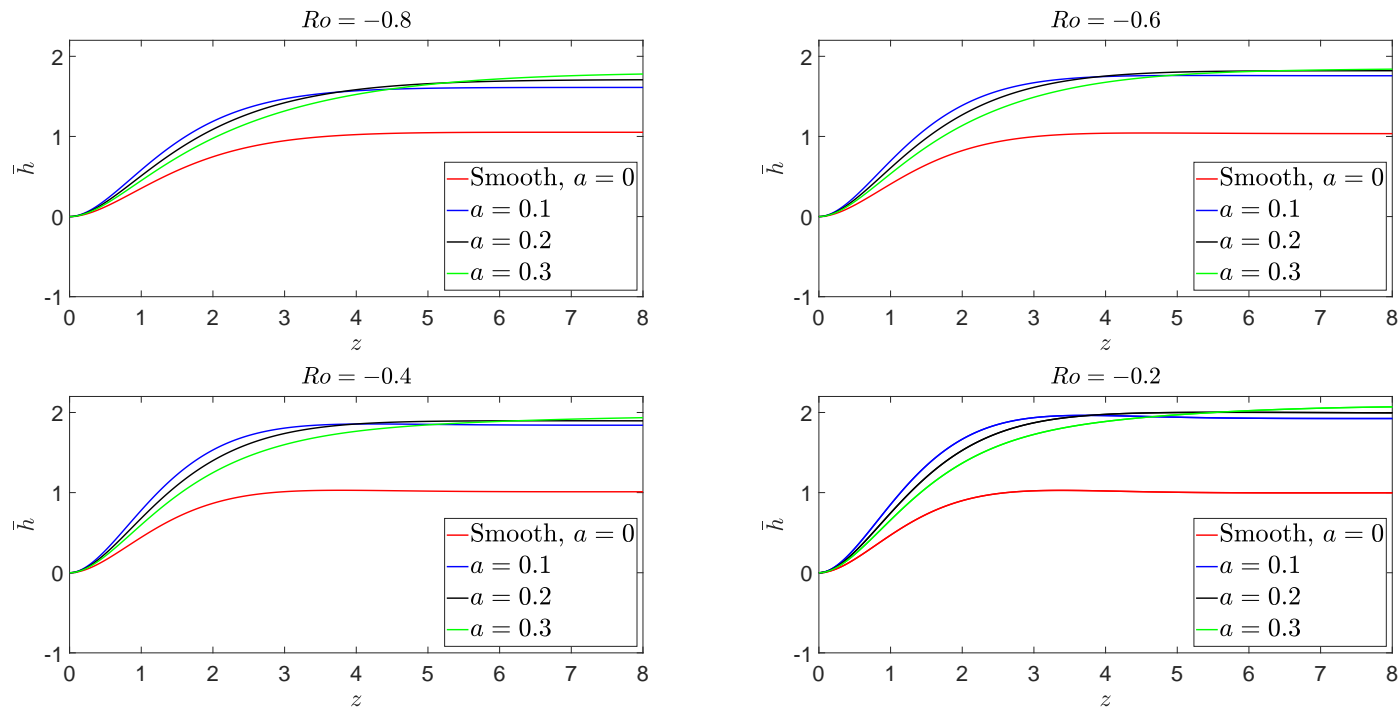

Figure 3.3: The mean flow components in axial direction at various values of roughness parameter $a$. 
The spatially averaged flow components of the YHP model over one wavelength for roughness parameter $a=0$ to $a=0.3$ are given in Figures 3.1-3.3. The results indicate that increasing roughness level reduces the amount of the wall jet at vicinity of disk surface, i.e., it decreases the maximum radial velocity, $\max (\bar{f})$, as seen in Figure 3.2(a)-(d) for each flow configuration. Also, increased roughness leads to an incremental widening of the azimuthal velocity component as shown in Figure 3.2(a)-(d) for each flow. In other words, increased roughness makes the boundary layer thicker. However, Figure 3.3(a)-(d) presents the increase in the magnitude of $\bar{h}$ component, i.e., the amount of the axial flow increases with the greater values of the roughness.

The MW and YHP roughness models are theoretically different as the latter one uses transformed coordinates. Therefore, it is not possible to make a quantitative comparison for equal values of roughness parameters. Instead, a qualitative approach is considered to compare the effects of increased roughness levels for both models. The moderate roughness value $a=0.2$ is selected in YHP model and the maximum values of radial jets of flows between $-1<R o<0$ are matched for both models. In other words, for each flow, the corresponding roughness parameter value of $\eta$ in the MW model is determined such that the maximum values of radial mean flow components are same under both models. The matched mean flow profiles of the different flows are presented in Figure 3.4(a)-(d). The numerical values of matching parameters are also presented at Table 1.

\begin{tabular}{|c|c|c|}
\hline Rossby Number $R o$ & $\max (\bar{f})$ & Roughness parameter $\eta$ for MW Model \\
\hline$R o=-0.8$ & -0.1528 & 0.988 \\
$R o=-0.6$ & -0.1813 & 0.689 \\
$R o=-0.4$ & -0.2030 & 0.521 \\
$R o=-0.2$ & -0.2220 & 0.416 \\
\hline \hline
\end{tabular}

Table 1: Corresponding matching values of different flows for $a=0.2$ of YHP model

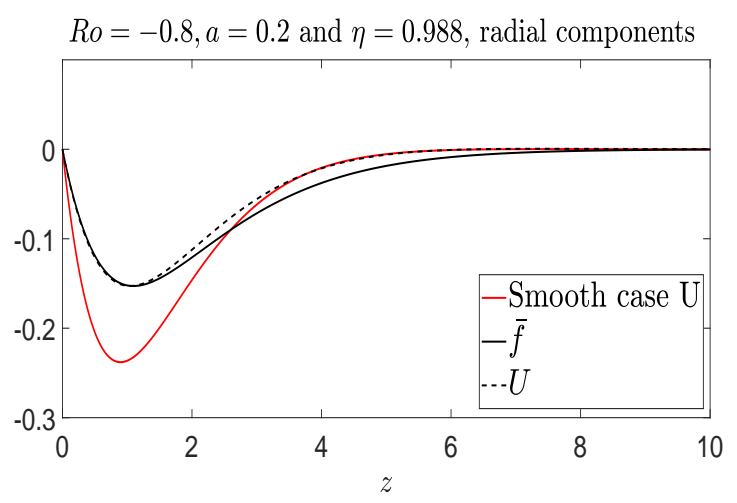

(a) $R o=-8$

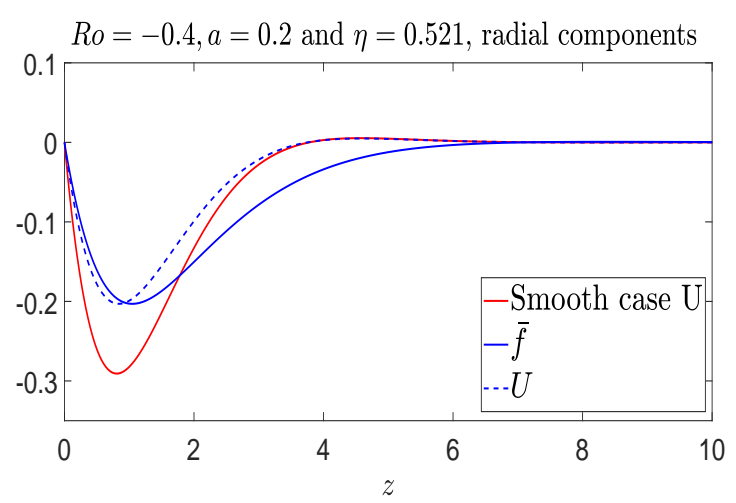

(c) $R o=-0.4$

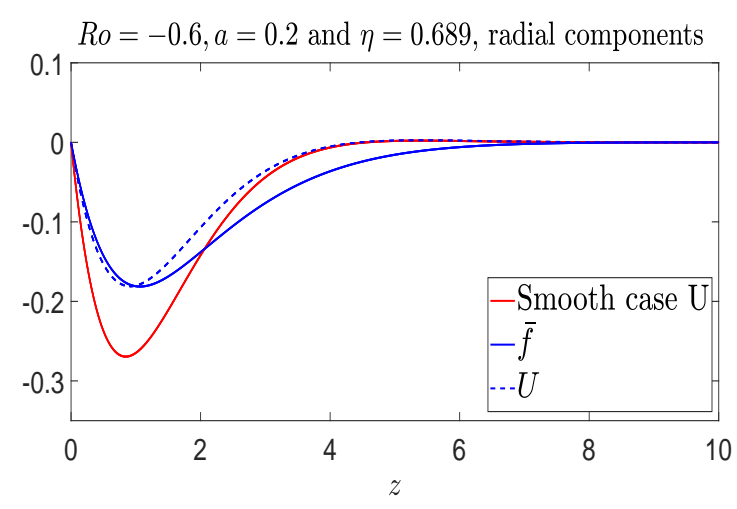

(b) $R o=-0.6$

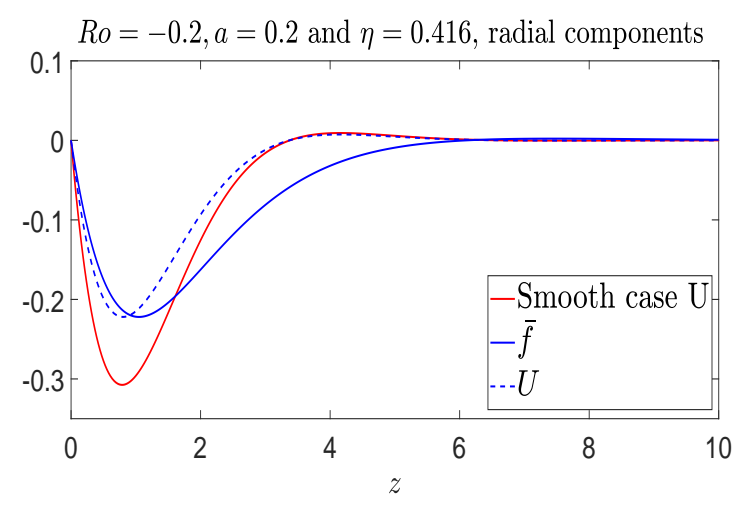

(d) $R o=-0.2$

Figure 3.4: Compared mean flow profiles of different flows in case of the MW and YHP models in radial direction. 


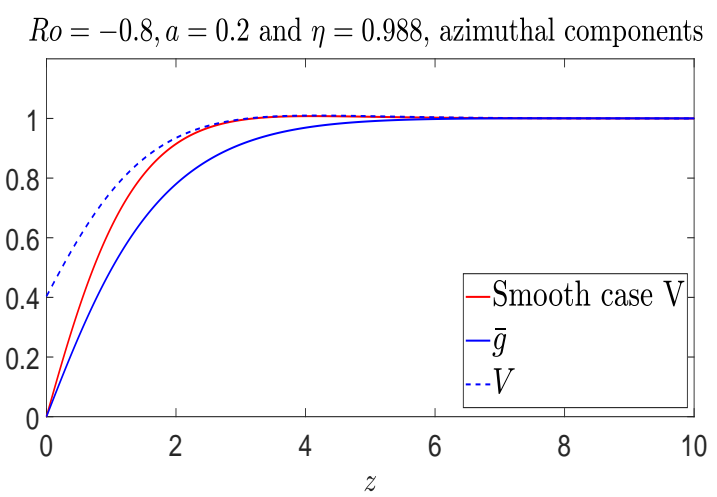

(a) $R o=-8$

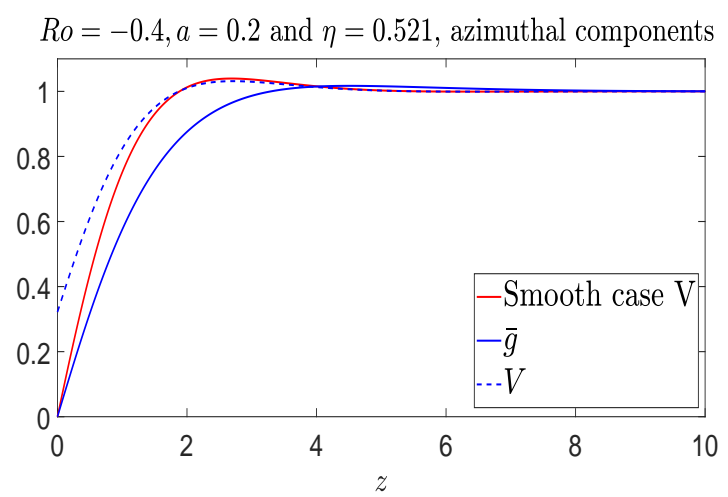

(c) $R o=-0.4$

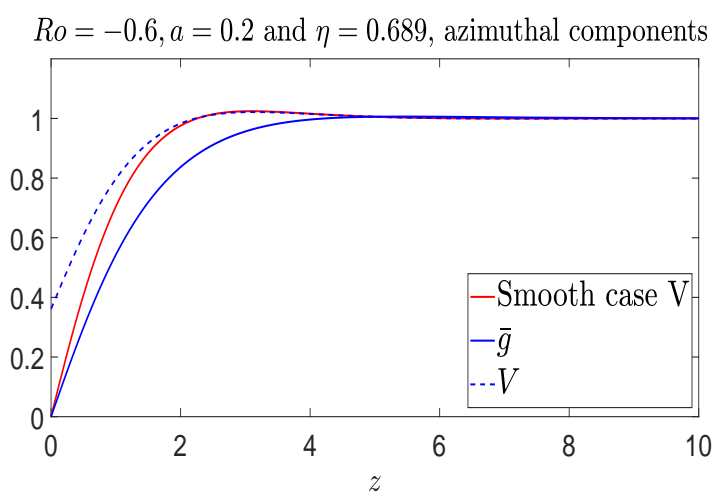

(b) $R o=-0.6$

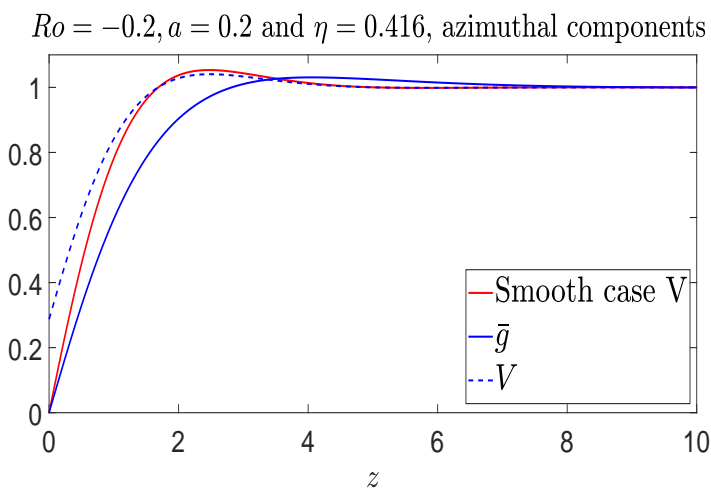

(d) $R o=-0.2$

Figure 3.5: Compared mean flow profiles of different flows in case of the MW and YHP models in azimuthal direction.

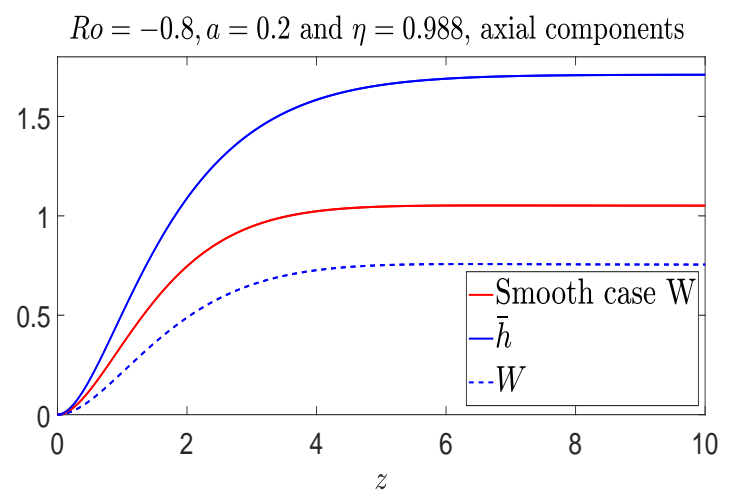

(a) $R o=-8$

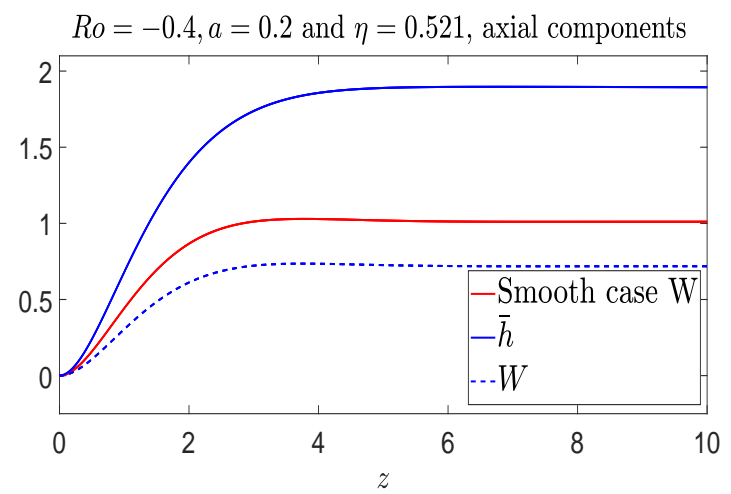

(c) $R o=-0.4$

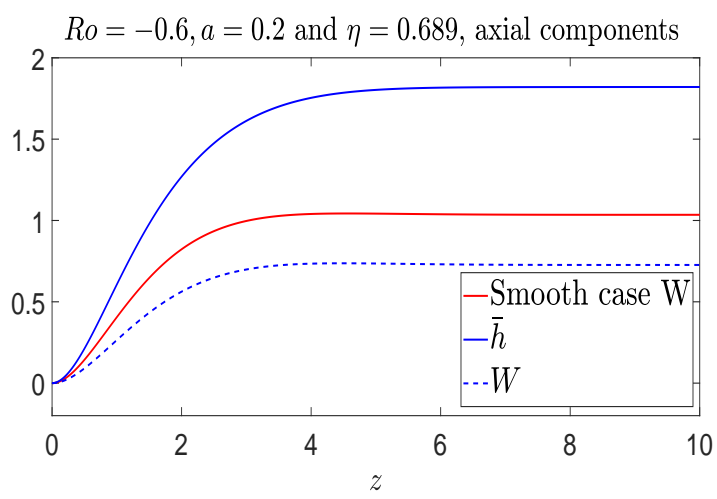

(b) $R o=-0.6$

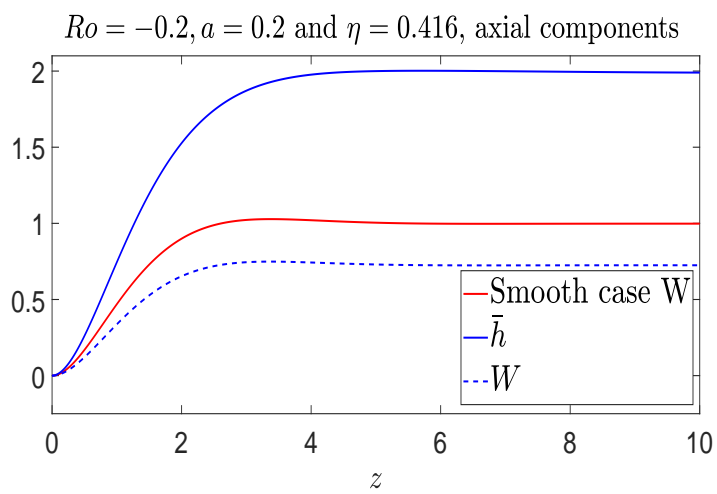

(d) $R o=-0.2$

Figure 3.6: Compared mean flow profiles of different flows in case of the MW and YHP models in axial direction. 
The difference of the effects of the MW and YHP models can be interpreted from Figures 3.5 - 3.6. The solid red lines in each parts shows the velocity components in absence of roughness. Figure 3.5 includes the velocity profiles in azimuthal direction and reveals that applying the MW model increases the value of azimuthal velocity profile for each flow, whereas applying YHP model causes a decrease. However, this difference is not huge and the values of this component become equal for each model at the far field of $z$ domain. The main different effect of the models, on the other hand, has been observed at the axial flow components of each flow. The results for these components, $\bar{h}$ in the YHP model and $W$ in the MW model, are represented in Figure 3.6. This figure reveals a substantial increase in the amount of axial flow in case of the YHP model, and a reduction in case of the MW model. Moreover, the presence of the roughness modelled with the YHP model seems to increase oscillatory behaviour of that component compared to the smooth case.

\section{Conclusion}

The aim of this study was to investigate the effects of the YHP model [15] in a rotating frame of reference on the mean flow solutions of some member of the BEK system flows, particularly the flows with $-1<R o<0$. The mean flow profiles are computed using the method of lines and the backward difference from highly nonlinear governing equations (2.2). The obtained mean-flow components agree with the previous findings in case of a smooth disk [4, 11]. The results indicate that increased roughness for each flow vanishes the oscillations of the steady mean flows towards the boundary layer, and the magnitude of the wall jet, $\bar{f}$, is also reduced.

The computed profiles are also compared with the results of another theoretical roughness model, the MW model [14]. The comparison is made in each flow for selected the roughness parameter value $a=0.2$ of YHP model and the corresponding roughness parameter $\eta$ of the MW model. It seems that the results are changed substantially due to selection of the roughness model. This points possible future researches investigating the effects of the roughness models on the stability characteristics of the flows.

\section{Acknowledgement}

Author would like to thank the reviewers for their thoughtful comments and efforts towards improving this manuscript.

\section{References}

[1] W. E. Gray, The nature of the boundary layer flow at the nose of a swept wing, Roy. Aircraft Est. TM, 256 (1952).

[2] D. Poll, Some observations of the transition process on the windward face of a long yawed cylinder, J. Fluid Mech., 150 (1985), $329-356$.

[3] P. Hall, An asymptotic investigation of the stationary modes of instability of the boundary layer on a rotating disc, Proc. R. Soc. Lond. Ser. A Math. Phys. Eng. Sci., 406 (1986).

[4] R. J. Lingwood, Absolute instability of the ekman layer and related rotating flows, J. Fluid Mech., 331 (1997), $405-428$.

[5] P. Carpenter, The right sort of roughness, Nature, 388(6644) (1997), 713-714.

[6] K. Choi, Fluid dynamics: The rough with the smooth, Nature, 440(7085) (2006), 754-754.

[7] L. Sirovich, S. Karlsson, Turbulent drag reduction by passive mechanisms, Nature, 388(6644) (1997), $753-755$.

[8] A. J. Colley, P. J. Thomas, P. W. Carpenter, A. J. Cooper, An experimental study of boundary-layer transition over a rotating, compliant disk, Phys. Fluids (1994-present), 11(11) (1999), 3340-3352.

[9] Garrett, S. J. and Cooper, A. J. and Harris, J. H. and Ozkan, M. and Segalini, A. and Thomas, P. J., On the stability of von Kármán rotating-disk boundary layers with radial anisotropic surface roughness. Physics of Fluids, 28 (2016), 014104

[10] T. Watanabe, H. M. Warui, N. Fujisawa, Effect of distributed roughness on laminar-turbulent transition in the boundary layer over a rotating cone, Experiments Fluids, 14(5) (1993), 390-392.

[11] Alveroğlu, B. and Segalini, A. and Garrett, S. J., The effect of surface roughness on the convective instability of the BEK family of boundary-layer flows, European Journal of Mechanics-B/Fluids, 56 (2016), 178-187.

[12] A. J. Cooper, J. H. Harris, S. J. Garrett, M. Ozkan, P. J. Thomas, The effect of anisotropic and isotropic roughness on the convective stability of the rotating disk boundary layer, Phys. Fluids, 27(1) (2015), 16.

[13] A. J. Cooper, P. W. Carpenter, The stability of rotating-disc boundary-layer flow over a compliant wall. part 1. type I and II instabilities, J. Fluid Mech., 350 (1997), 231-259.

[14] M. Miklavcic, C. Y. Wang, The flow due to a rough rotating disk, Z. Angew. Math. Phys., 55(2) (2004), $235-246$.

[15] M. S. Yoon, J. M. Hyun, P. Jun Sang, Flow and heat transfer over a rotating disk with surface roughness, Int. J. Heat Fluid Flow, 28(2) (2007), 262-267. 